\title{
Probing Polar Molecules with High Harmonic Spectroscopy
}

\author{
E. Frumker, ${ }^{1,2,3, *}$ N. Kajumba, ${ }^{1}$ J. B. Bertrand, ${ }^{1}$ H. J. Wörner, ${ }^{1}$ C. T. Hebeisen, ${ }^{1}$ P. Hockett, ${ }^{4}$ M. Spanner, ${ }^{4}$ \\ S. Patchkowskii, ${ }^{4}$ G. G. Paulus, ${ }^{3}$ D. M. Villeneuve, ${ }^{1}$ A. Naumov, ${ }^{1}$ and P. B. Corkum ${ }^{1, \dagger}$ \\ ${ }^{1}$ Joint Attosecond Science Laboratory, University of Ottawa and National Research Council of Canada, \\ 100 Sussex Drive, Ottawa, Ontario K1A 0R6, Canada \\ ${ }^{2}$ Max-Planck-Institut für Quantenoptik, Hans-Kopfermann-Strasse 1, D-85748 Garching, Germany \\ ${ }^{3}$ Department of Physics, Texas A\&M University, College Station, Texas 77843, USA \\ ${ }^{4}$ Steacie Institute for Molecular Sciences, National Research Council of Canada, Ottawa, Ontario K1A OR6, Canada
} (Received 25 July 2012; published 4 December 2012)

\begin{abstract}
We bring the methodology of orienting polar molecules together with the phase sensitivity of high harmonic spectroscopy to experimentally compare the phase difference of attosecond bursts of radiation emitted upon electron recollision from different ends of a polar molecule. This phase difference has an impact on harmonics from aligned polar molecules, suppressing emission from the molecules parallel to the driving laser field while favoring the perpendicular ones. For oriented molecules, we measure the amplitude ratio of even to odd harmonics produced when intense light irradiates $\mathrm{CO}$ molecules and determine the degree of orientation and the phase difference of attosecond bursts using molecular frame ionization and recombination amplitudes. The sensitivity of the high harmonic spectrum to subtle phase differences in the emitted radiation makes it a detailed probe of polar molecules and will drive major advances in the theory of high harmonic generation.
\end{abstract}

DOI: 10.1103/PhysRevLett.109.233904

PACS numbers: 42.65.Ky, 07.57.-c

High harmonic generation is a sub-optical-cycle process. During successive half-periods, an electron wave packet recollides with its parent ion from alternate directions producing successive attosecond bursts of extreme ultraviolet radiation [1]. If a symmetric molecule is the nonlinear medium, then this periodicity produces odd harmonics because successive bursts are identical except for a $\pi$ phase difference.

A polar molecule, on the other hand, looks different depending on the direction in which it is viewed. When high harmonics are generated from polar molecules, alternate attosecond bursts have different amplitude and phase. Thus, molecular asymmetry is embedded in the single molecule response and in the phase-matched spectrum that we measure (as illustrated in Fig. 1). Asymmetry is imposed at each step of the harmonic generation process [1]: when the electron wave packet is detached from the target molecule; when the liberated electron gains energy and phase in the continuum as it accelerates away from the parent ion and then is driven back to recollide; and when the electron recombines with the parent ion.

Building on the first demonstration of high harmonic generation with aligned and oriented molecules in [2,3], we now report on a new approach for probing the structure of polar molecules. We use both the techniques of laser alignment [4] (up-down symmetric with respect to the aligning laser field [5]) and two-color laser-orientation [6] (broken up-down symmetry) of molecules to make high harmonic generation measurements in the aligned as well as the oriented molecular frame. We confirm that an oriented rotational wave packet is produced in $\mathrm{CO}$ by probing with
Coulomb explosion imaging [7]. These measurements are repeated on aligned $\mathrm{N}_{2}$, which serves as an isoelectronic nonpolar reference for comparison. Their orbital structure, their angle-dependent ionization probability, and their transition moments are all shown in Fig 2. Our experiment shows how information on the polar molecule is encoded in both the odd harmonic spectrum of aligned molecules and in the even harmonics of oriented molecules.

We implement the two-color method of laser-induced orientation [8,9] for high density gas [3]. Early studies on two-color orientation considered only the hyperpolarizability mechanism [9] of laser-generated orientation. As discussed in full elsewhere [3,10], we believe an ionization depletion mechanism is active in our experiment. Briefly, the molecular ionization probability of polar molecules is sensitive to the orientation between the molecular frame and the ionizing laser polarization $[11,12]$. Therefore, in a maximally asymmetric electric field produced by adding a pulse with frequency $\omega$ to a second-harmonic pulse at $2 \omega$, ionization will partially deplete the neutral population of an initially randomly oriented sample at certain orientations, while leaving molecules in their neutral state at other orientations. The orientation-dependent ionization creates an oriented molecular distribution in both the neutral molecule and in the ion and the same laser pulse launches the rotational molecular wave packet. In the unionized (ground state) the molecular ensemble is transiently aligned and oriented during the rotational wave packet revivals.

Either $\mathrm{N}_{2}$ or $\mathrm{CO}$ [ionization potential $(I P)=15.6$ and $14.0 \mathrm{eV}$, respectively, and dipole moment $\left(\mathrm{D}_{\mathrm{CO}}\right)=0.112$ Debye] [13] was separately injected into a vacuum system 


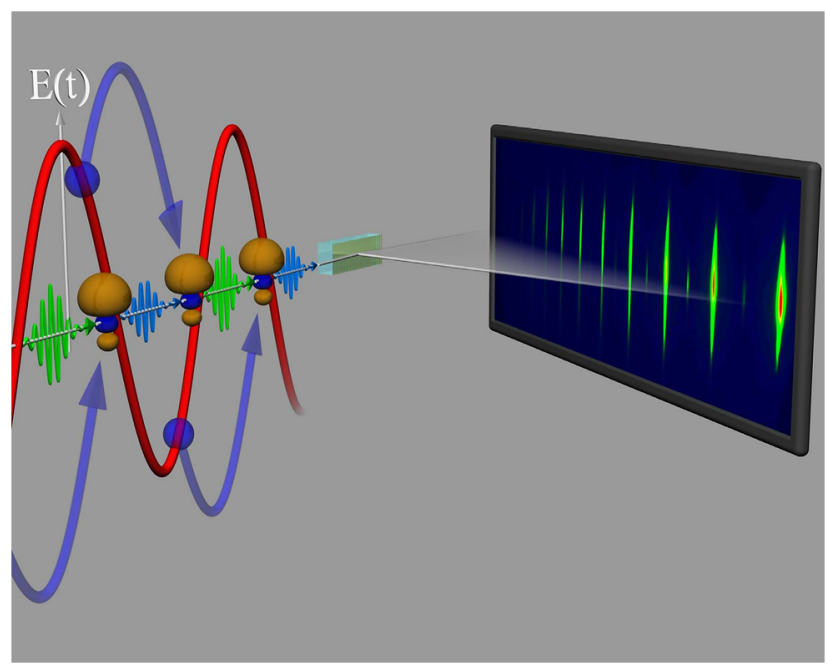

FIG. 1 (color online). Spectral interference lies at the heart of high harmonic generation from oriented molecules. Every 1/2 period of the fundamental intense infrared laser pulse (shown in red), an electron wave packet is detached from an oriented molecule, oscillates in the time-dependent electric field and recombines to create an attosecond burst of XUV light. An example of four such bursts of a train is shown in the figure. In our experiment, this attosecond pulse train is diffracted and imaged by a grating. The spectral interference of such a train results in the formation of even and odd harmonics. An experimental spectrum is shown in the figure.

using a supersonic jet. Experiments have shown that they have similar ionization probabilities when averaged over all orientations $[14,15]$. We achieve alignment and orientation using a pulse of $800 \mathrm{~nm}$ light and its second harmonic $(\omega+2 \omega)$. After a variable delay, we probe the rotational wave packet by generating high harmonics with an intense $800 \mathrm{~nm}$ pulse incident at an angle to the pump beam so that any harmonics created by the probe are spatially separated from those created by the pump beam. By delaying the probe, we observe the neutral rotational wave packets at different stages of alignment and orientation. The full optical setup is described elsewhere [3].

Figure 3 (upper panel) shows the time evolution of a typical odd harmonic $\left(\mathrm{H} 19 ; h \nu=29 \mathrm{eV}\right.$ ) for $\mathrm{N}_{2}$ (dashed blue line) and $\mathrm{CO}$ (solid red line). The origin of the time axis for both molecules is taken as the time of the first full rotational revival $(\tau=\pi / B$ where $B$ is the rotational constant). For comparison, the dotted green curve in Fig. 3 (upper panel) shows a nonoriented rotational wave packet of $\mathrm{CO}$ as measured by Coulomb explosion imaging [7]. $\mathrm{CO}$ is parallel to the laser field at the time of the H19 minimum. In contrast, the harmonic signal maximizes when $\mathrm{N}_{2}$ is parallel [16]. This contrasting behavior between $\mathrm{N}_{2}$ and $\mathrm{CO}$ persists for all harmonics that we have studied except for the very lowest (H11 and H13).

There are two possible reasons for this contrasting behavior. First, the orbital structure of $\mathrm{CO}$ and $\mathrm{N}_{2}$ allows the HOMO-1 orbital to contribute when the molecule is (a)

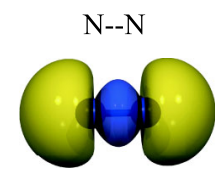

(b)

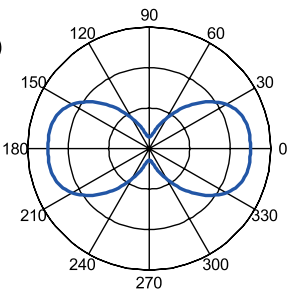

(c)
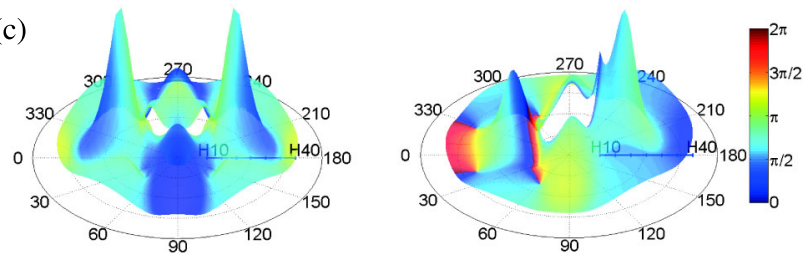

FIG. 2 (color online). (a) The Dyson orbital for the most weakly bound valence electron of the isoelectronic pair, $\mathrm{N}_{2}$ and CO. The color code represents a $\pi$ phase difference. The orientation is preserved in (b) and (c). (b) The total ionization yield as a function of the angle between the molecular axis and the electrical field calculated for a $1 / 2$ cycle of $800 \mathrm{~nm}$ with intensity $1.5 \times 10^{14} \mathrm{~W} / \mathrm{cm}^{2}$. (c) The amplitude (vertical axis) and phase (color code) of the transition moment as a function of angle and harmonic order (radial axis) for XUV light polarized along the molecular axis.

perpendicular. This enhances the high harmonic yield for perpendicular molecules [17].

Second, when the laser polarization is along the direction of asymmetry, successive attosecond bursts are quite different. The complex spectral amplitude of the emitted radiation within a single period of the fundamental field is given by $\tilde{E}(\nu)=\tilde{E}_{1}(\nu)-\tilde{E}_{2}(\nu) e^{-i \pi \nu T}$, where $\tilde{E}_{1}(\nu)$ and $\tilde{E}_{2}(\nu)$ are spectral complex amplitudes of the attosecond bursts emitted from opposite sides of the polar molecule, $\nu$ is the frequency of the emitted radiation, and $T$ is the period of the driving laser field. The phase factor $e^{-i \pi \nu T}$ accounts for the fact that the second burst $-E_{2}(t)$ is shifted in time by a half period $T / 2$ relative to the first one $-E_{1}(t)$.

When the driving laser field is composed of multiple cycles the even and odd harmonics are formed with the respective complex spectral amplitudes given by $\tilde{E}_{\text {even }}[2 n / T]=\tilde{E}_{1}[2 n / T]-\tilde{E}_{2}[2 n / T]$, and $\tilde{E}_{\text {odd }}[(2 n-1) / T]=\tilde{E}_{1}[(2 n-1) / T]+\tilde{E}_{2}[(2 n-1) / T]$ with $n=1,2, \ldots$. The phase and/or amplitude differences (beyond the normal $\pi$ phase difference for symmetric systems) between subsequent attosecond bursts lead to even harmonic formation at the single molecule level, but it also reduces the odd harmonic response [18] since maximal constructive interference of the odd harmonics does not, in general, occur. As already discussed (Fig. 1), this cycleto-cycle spectral interference is at the heart of harmonic 


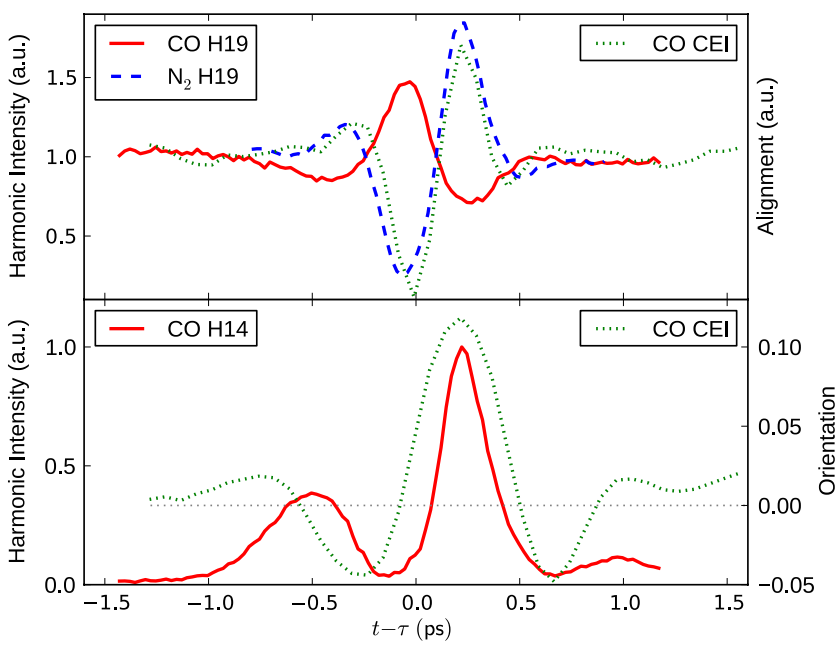

FIG. 3 (color online). Upper plot: Dotted green curve shows alignment of $\mathrm{CO}$ determined from a Coulomb explosion imaging measurement. The time-resolved high harmonic yields (19th harmonic), corresponding to the same rotational wave packet, are shown for $\mathrm{CO}$ (solid red curve) and $\mathrm{N}_{2}$ (dashed blue curve) near the time of their first full revival (set at $t=0$ ). Lower plot: An orientational wave packet in $\mathrm{CO}$ excited by the superposition of 800 and $400 \mathrm{~nm}$ light. Dotted green curve shows the orientation of CO measured by Coulomb explosion imaging. The red solid curve shows the time dependent signal for the 14th harmonic. Note that the even harmonic signal is maximum when the orientation is greatest.

generation with polar molecules and the reason for its inherent sensitivity to molecular asymmetries.

The emission of odd harmonic signal is independent of the degree of orientation [18]; thus, the reduction of the odd harmonic signal persists, even when the sample as a whole is symmetric. In other words, the angle dependence of the odd harmonic signal carries information on a polar molecule. A simulation of parallel and perpendicular odd harmonics for $\mathrm{N}_{2}$ and $\mathrm{CO}$ based on HOMO orbitals exclusively is presented in the Supplemental Material [19]. We will return to this issue later in the Letter.

The time-dependent orientation of $\mathrm{CO}$, as determined by Coulomb explosion imaging, is plotted in the lower panel of Fig. 3 (dashed green curve). Orientation is measured by the cosine of the angle between an axis parallel to the electric field of the two-color laser pulse and the momentum (or inverse momentum) of the $\mathrm{C}^{++}$(or $\mathrm{O}^{++}$) fragments. The time dependence of a typical even harmonic (H14; $h \nu=22 \mathrm{eV}$; solid red line) maximizes when the orientation maximizes. The even harmonics appear on zero background, allowing a signal-to-noise of better than 500:1.

Figure 4 shows the harmonics spectrum taken at a pumpprobe time delay of $8.85 \mathrm{ps}$ - the time of maximum orientation. Even harmonics are visible between the stronger odd harmonics. To ensure the greatest possible linearity of the detector and to facilitate extended dynamic range data

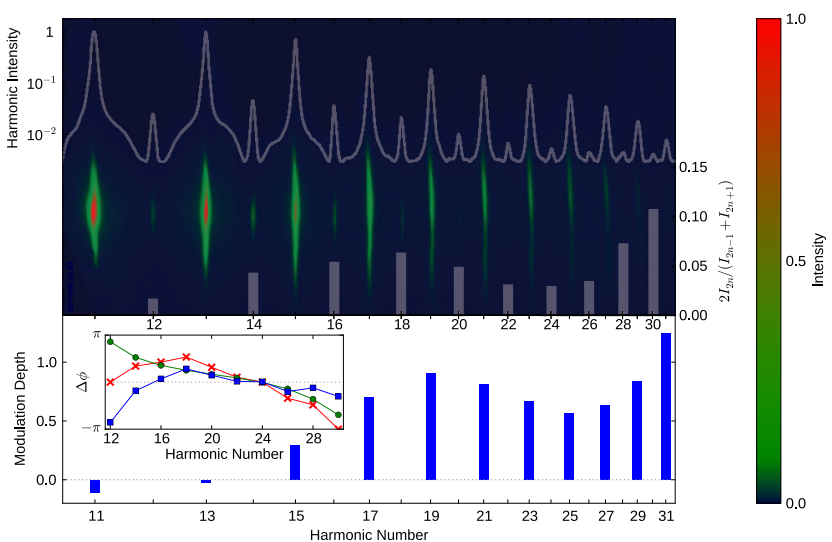

FIG. 4 (color online). The high harmonic spectrum is shown at $8.85 \mathrm{fs}$, the time of maximum orientation. The intensity of all harmonics is presented in the top panel of the figure, showing in graphical form what is measured in the image below. The ratio of even to the average of the adjacent odd harmonics is shown in the upper bar graph. The lower bar graph plots the normalized difference $-R=\left(S_{a a}-S_{a}\right) / S_{b}$. The figure inset shows the phase difference (in green, circle marks) for an electron departing from the $-\mathrm{C}$ - side and the $-\mathrm{O}$ - side of the molecule as determined by $e$-polyscat simulations. The red (cross marks) curve is the measured spectral phase asymmetry of successive attosecond XUV (recollision electron) pulses (relative to the normally expected asymmetry). The blue (square marks) curve is the recolliding electron wave-packet spectral phase asymmetry.

acquisition, the microchannel plate detector was operated at a low voltage $(1700 \mathrm{~V}$ across the microchannel plate chevron pair), and the same image was acquired by the cooled charge-coupled device multiple times with different integration times. We determined the linear range of the camera with respect to the integrated intensity and masked out saturated and nonlinear regions of each image. The remaining parts of the images were integrated and calibrated according to the total acquisition time of each pixel. This method provides substantial noise reduction and an extended dynamic range over single acquisitions.

In Fig. 4, the upper curve is the integrated signal of the experimental image. The ratio of intensities of each even harmonic to the average of the adjacent odd harmonic orders is plotted as the upper of the two bar graphs. The ratio increases up to $h \nu=28 \mathrm{eV}$ (H18). It has a clear minimum around a photon energy of $37 \mathrm{eV}$ (H24) and then grows monotonically to the cutoff. In the lower panel of the same figure the ratio $R=\left(S_{a a}-S_{a}\right) / S_{b}$ is plotted for the odd harmonics from a rotational wave packet. Here, $S_{a a}, S_{a}$, and $S_{b}$ represent the harmonic signal for aligned (a), antialigned (aa), and baseline (b) molecular axis distributions. We measure $S_{b}$ after the revival has dephased. The similarity of the two bar graphs, measuring seemly different quantities-alignment dynamics and even to odd harmonic ratios-is striking.

Along with the phase asymmetry, the measurement of the ratio of even to the average of the adjacent odd harmonics 
allows us to estimate the degree of orientation in our experiments as described in Section B of the Supplemental Material [19]. We find the degree of orientation to be $\alpha_{0}=$ 0.24 consistent with the estimate of 0.2 in Ref. [3].

To progress further we follow each step of the three-step model of high harmonic generation [1].

Tunneling.-On adjacent crests of the electric field, an electron wave packet is launched with a different amplitude and phase. The calculated molecular frame ionization probability is shown in Fig. 2(b), right column. Amplitude ratios $r_{t}=A_{t}^{O} / A_{t}^{C}$ of the electron wave packets created by tunneling have been measured for a few polar diatomic molecules $[14,15]$. Here $\mathrm{O}$ represents the oxygen side of the molecule and $\mathrm{C}$ the carbon side. As discussed earlier, it is this significant difference in tunneling probability shown in Fig. 2(b) which facilitates the creation of an oriented sample via depletion $[3,10]$.

Wave packet propagation.-Once the electron wave packet is launched into the continuum by tunneling, phase accumulates rapidly relative to the Stark-shifted ion ground state. Asymmetry primarily arises because of the different Stark shift the molecule experiences when the field is parallel or antiparallel to the dipole moment of the molecule and molecular ion [20]. While propagation will have little impact on the amplitude asymmetry of the attosecond burst, phase difference $\delta \phi_{p}$ will strongly depend on the harmonic order.

Recombination.-The final step describes the electron wave packet recombining to its initial bound state. Quantum chemistry codes are well developed for calculating field-free dipole transition moments [21,22], shown in Fig. 2(c) for CO. Both amplitude (plotted in the third dimension) and phase (represented by color) depend on the photoelectron's direction relative to the molecule leading to an amplitude ratio $r_{r}$ and a phase difference $\delta \phi_{p}$. The field-free transition moment amplitudes have been used successfully to describe high harmonic generation from atoms and small molecules [23,24].

Using the amplitude ratios shown in Figs. 2(b) and 2(c) and the experimentally estimated degree-of-orientation [19] we can estimate the electric field amplitude asymmetry of the attosecond bursts. With this ratio known, the measured spectrum allows determination of the phase difference between successive attosecond XUV pulses as shown in the Supplemental Material [19]. The reconstructed phase difference of the attosecond pulses, $\delta \phi_{A}$ is plotted in the inset in Fig. 4 as the red curve (the $\pi$-phase difference that arises from the oppositely directed recollision on each half cycle has been subtracted for clarity).

We are now in a position to return to the normalized difference for the odd harmonics (lower panel of Fig. 4). We have just seen that the phase difference of successive XUV pulses is $\pi$ near $h \nu=37 \mathrm{eV}$ (H25). Therefore, the successive attosecond pulses contribute optimally to the odd harmonics of the aligned molecule near $\mathrm{H} 25$ just as they do for all frequencies for antialigned molecules. While other aspects could contribute, we should not be surprised to see a minimum in the modulation depth $R=$ $\left(S_{a a}-S_{a}\right) / S_{b}$ (Fig. 4 bottom panel). In other words, the minimum in the normalized difference and even or odd harmonic ratio probably shares a common origin because $\delta \phi_{A}=0$ at $\mathrm{H} 25(37 \mathrm{eV})$.

The high harmonic spectra from oriented polar molecules poses challenges for theoretical models since, unlike the case for high harmonic generation from homogeneous samples, the calculation of the emitted spectra demands that the subtle phase asymmetries present in each step be determined accurately. Subcycle measurements will make this and other phase differences experimentally accessible parameters. The three-step model requires that $\delta \phi_{A}=\delta \phi_{t}+\delta \phi_{p}+$ $\delta \phi_{r}+\pi$ where the three terms represent the phase difference the electron acquires on tunneling, propagation, and recombination. To show the potential impact of measurements on polar molecules, we use the recombination phase from quantum chemistry codes to evaluate $\delta \phi_{r}$ [shown in Fig. 2(c), right column, and replotted as the green curve with circle marks in the inset in Fig. 4]. With the recombination phase removed, the sum $\delta \phi_{t}+\delta \phi_{p}+\pi$ is the phase asymmetry of the attosecond electron wave packet at the moment of recollision. This phase of the recollision electron wave packet will be experimentally accessible in abovethreshold ionization experiments [25]. We could estimate the propagation phase $\delta \phi_{p}$, determining the unknown tunneling phase, but the meaning of this parameter would require a special discussion. Future theory and experiments will allow us to zero in on a well-defined tunneling phase.

In conclusion, by orienting high density samples of molecules we have shown how the coherence of high harmonic spectroscopy provides a new, powerful tool for probing asymmetries of polar molecules. In carefully designed experiments, subcycle physics [26] will give us access to all parameters. The phase differences that will become accessible arise, in part, from subtle differences in the local fields and from the quantum exchange interaction that the electron experiences when it is born and when it recombines. Thus, polar molecules will drive major advances in the theory of high harmonic generation while high harmonic spectroscopy will yield unprecedented insight into polar molecules and into multielectron physics.

We acknowledge financial support of Canada's National Research Council, National Sciences and Engineering Research Council, the Canada Research Chair Program and MURI Grant No. W911NF-07-1-0475. E. F acknowledges the support of Marie Curie International Outgoing Fellowship.

*eugene.frumker@mpq.mpg.de

†paul.corkum@nrc.ca

[1] P. B. Corkum, Phys. Rev. Lett. 71, 1994 (1993). 
[2] E. Frumker, N. Kajumba, J. B. Bertrand, H. J. Worner, C. T. Hebeisen, P. Hockett, M. Spanner, S. Patchkowskii, G. G. Paulus, D.M. Villeneuve, and P.B. Corkum, in Proceedings of the 3rd International Conference on Attosecond Physics, Sapporo, Japan, 2011, http://www.icomp-atto.org/ATTO/ contents/contributed.html; arXiv:1205.4108.

[3] E. Frumker, C. T. Hebeisen, N. Kajumba, J. B. Bertrand, H. J. Worner, M. Spanner, D. M. Villeneuve, A. Naumov, and P. B. Corkum, Phys. Rev. Lett. 109, 113901 (2012).

[4] O. Ghafur, A. Rouzee, A. Gijsbertsen, W. K. Siu, S. Stolte, and M. J. J. Vrakking, Nat. Phys. 5, 289 (2009).

[5] H. Stapelfeldt and T. Seideman, Rev. Mod. Phys. 75, 543 (2003).

[6] A. Goban, S. Minemoto, and H. Sakai, Phys. Rev. Lett. 101, 013001 (2008).

[7] P. W. Dooley, I. V. Litvinyuk, K. F. Lee, D. M. Rayner, M. Spanner, D. M. Villeneuve, and P. B. Corkum, Phys. Rev. A 68, 023406 (2003).

[8] H. Sakai, S. Minemoto, H. Nanjo, H. Tanji, and T. Suzuki, Phys. Rev. Lett. 90, 083001 (2003).

[9] S. De, I. Znakovskaya, D. Ray, F. Anis, Nora G. Johnson, I. A. Bocharova, M. Magrakvelidze, B.D. Esry, C. L. Cocke, I. V. Litvinyuk, and M. F. Kling, Phys. Rev. Lett. 103, 153002 (2009).

[10] M. Spanner, S. Patchkovskii, E. Frumker, and P. B. Corkum. Phys. Rev. Lett. 109, 113001 (2012).

[11] H. Akagi, T. Otobe, A. Staudte, A. Shiner, F. Turner, R. Doerner, D. M. Villeneuve, and P. B. Corkum, Science 325, 1364 (2009).

[12] L. Holmegaard, J. L. Hansen, L. Kalhoj, S. L. Kragh, H. Stapelfeldt, F. Filsinger, J. Kuepper, G. Meijer, D. Dimitrovski, M. Abu-samha, C. P. J. Martiny, and L. B. Madsen, Nat. Phys. 6, 428 (2010).
[13] K. P. Huber and G. Herzberg, Molecular Spectra and Molecular Structure (Van Nostrand Reinhold, New York, 1979).

[14] D. Pinkham and R. R. Jones, Phys. Rev. A 72, 023418 (2005).

[15] A. S. Alnaser, C. M. Maharjan, X. M. Tong, B. Ulrich, P. Ranitovic, B. Shan, Z. Chang, C. D. Lin, C. L. Cocke, and I. V. Litvinyuk, Phys. Rev. A 71 (2005).

[16] J. Itatani, D. Zeidler, J. Levesque, M. Spanner, D. M. Villeneuve, and P. B. Corkum, Phys. Rev. Lett. 94, 123902 (2005).

[17] B. K. McFarland, J. P. Farrell, P. H. Bucksbaum, and M. Guhr, Science 322, 1232 (2008).

[18] E. Frumker, "Attosecond Burst Interference in High-Harmonic Generation with Polar Molecules" (unpublished).

[19] See Supplemental Material at http://link.aps.org/ supplemental/10.1103/PhysRevLett.109.233904 for some theoretical and numerical analysis.

[20] A. Etches and L. B. Madsen, J. Phys. B 43, 155602 (2010).

[21] F. A. Gianturco, R. R. Lucchese, and N. Sanna, J. Chem. Phys. 100, 6464 (1994).

[22] A. P. P. Natalense and R. R. Lucchese, J. Chem. Phys. 111, 5344 (1999).

[23] A. T. Le, R. R. Lucchese, S. Tonzani, T. Morishita, and C. D. Lin, Phys. Rev. A 80, 013401 (2009).

[24] A. D. Shiner, B.E. Schmidt, C. Trallero-Herrero, H. J. Wörner, S. Patchkovskii, P. B. Corkum, J.C. Kieffer, F. Légaré, and D. M. Villeneuve, Nat. Phys. 7, 464 (2011).

[25] Xinhua Xie, S. Roither, D. Kartashov, E. Persson, D. G. Arboacute, Li Zhang, S. Graumlfe, M. S. Schoumlffler, J. Burgdoumlrfer, A. Baltuscaronka, and M. Kitzler, Phys. Rev. Lett. 108, 193004 (2012).

[26] P. B. Corkum, Phys. Today 64, No. 3, 36 (2011). 